\title{
Medios de comunicación como espacios alternativos para la inclusión de subjetividades de género diversas en la escuela
}

\author{
Sara Lorena Galvis ${ }^{1}$; Olga Lucía Obando²
}

Recibido: Mayo 2017 / Evaluado: Febrero 2018 / Aceptado: Marzo 2018

Resumen. En este artículo se reflexiona sobre la construcción de espacios alternativos para la inclusión de las subjetividades de género diversas en la escuela a través de los medios de comunicación. En un diálogo interdisciplinario se recurre a aportes postmodernos feministas y de género, y a contribuciones desde las teorías de la comunicación. Asimismo, se abordan las tendencias de interpretación del fenómeno relacional sujeto, fenómenos comunicativos mediáticos, cultura y sociedad desde posturas tecnofóbicas y tecnofílicas multidisciplinarias.

El análisis se fundamenta en una serie de situaciones ocurridas en Colombia donde la propuesta de inclusión de subjetividades de género diversas en la escuela genera reacciones sociales y políticas significativas al adquirir un carácter anticonstitucional y contrario a los principios negociados en los Acuerdos de Paz.

Las propuestas identificadas en los medios de comunicación tales como la diversificación y naturalización de imaginarios de género, la implementación de una oferta de conocimiento informado y la motivación de procesos de toma de conciencia de género señalan posibles ambivalencias con respecto al potencial de los medios como elemento facilitador u obstaculizador de aperturas de reconocimiento y respeto a las diversidades de género. Dichas propuestas identificadas como estrategias potencializan procesos para aproximarse a las subjetividades de género, procesos que pueden ser útiles en contextos escolares y otros contextos sociales.

Palabras claves: Subjetividad de género; diversidades de género; medios de comunicación; inclusión; escuela.

\section{[en] Media as alternative spaces for the inclusion of diverse gender subjectivities in the school}

\begin{abstract}
This article reflects about the construction of alternative spaces through media for the inclusion of diverse gender subjectivities in the school. The theoretical contributions used are made from an interdisciplinary dialogue of postmodern feminists and gender theorists, and also by theories of communication. Likewise, the tendencies of interpretation of the relational phenomenon subject-communicative mediatic phenomena- culture and society are approached from technophobic and technophilic positions, produced from different disciplinary knowledges.

The analysis is based on a series of situations that occurred in Colombia where the proposal of inclusion of diverse gender subjectivities in the school generated significant social and political reactions by acquiring an unconstitutional character and being contrary to the principles negotiated in the Peace Accords.
\end{abstract}

\footnotetext{
$1 \quad$ Universidad del Valle, Colombia sara.galvis@correounivalle.edu.co

2 Universidad del Valle, Colombia olga.obando@correounivalle.edu.co
} 
The proposals identified in media indicates possible ambivalence regarding the potential of media as a facilitator or obstacle to the openness of recognition and respect for gender diversity. Unveiling the way some media through strategies such as: the diversification and naturalization of gender imaginaries, the implementation of a supply of informed knowledge and the motivation of processes of gender awareness, potentiates processes to approach gender subjectivities useful in school contexts and other social contexts.

Keywords: Gender subjectivity; gender subversion; media; inclusion; school.

Sumario: 1. Introducción. 2. Estrategias para la inclusión de la diversidad de género en los medios de comunicación. 3. Inclusión de la diversidad en el sector educativo y ciudadano. Conclusiones. Bibliografía.

Cómo citar: Galvis, S. L.; Obando, O. L. (2018). Medios de comunicación como espacios alternativos para la inclusión de subjetividades de género diversas en la escuela, Investigaciones feministas 9.1, 173-193.

\section{Introducción}

Reflexionar sobre la construcción de espacios alternativos para una inclusión de las Subjetividades de Género Diversas (en adelante SGD) en la escuela a través de los Medios de Comunicación (en adelante MC) se constituye en una tarea para los miembros del grupo multidiscliplinario de investigación del proyecto Concepciones, representaciones, discursos y debates sobre subjetividades de género diversa en contextos de conflicto. Una herramienta para la educación ciudadana para la paz ${ }^{3}$. El fenómeno de diversidades de género en su relación con la experiencia educativa emerge como generador de reacciones sociales y políticas conflictivas en tres escenarios (Congreso, escuela y sociedad civil). Estos hechos ocurridos en Colombia entre 2014 y 2016 convocaron al grupo de investigación a exponer una voz académica crítica frente a dichos eventos. Esta voz trata de comprender el fenómeno y comprometerse con el desarrollo de propuestas alternativas que posibiliten la superación de las situaciones de exclusión e inequidad de género en los diferentes ambientes sociales. Entre los hechos se abordan: el caso de un estudiante de secundaria que se suicida por sentirse víctima de acoso debido a su condición diversa de preferencia sexual; la acción de tutela presentada por la madre de la víctima contra la institución educativa y otras por violación de derechos fundamentales del hijo; la emisión de la Sentencia T-478 de la Corte Constitucional, que le demanda al Ministerio de Educación (en adelante MEN) garantías constitucionales para el cumplimiento de los derechos de libre desarrollo de la personalidad y de educación; las movilizaciones sociales y debates en ámbitos de la esfera política por la implementación de una propuesta liderada por el MEN para la inclusión de la diversidad de género y sexualidad en los ambientes escolares.

En mayo de 2014 en el colegio Gimnasio Castillo Campestre de Bogotá los estudiantes Sergio Urrego y Horacio ${ }^{4}$ hicieron pública una expresión sentimental por medio de un beso lo que generó una situación de escándalo en la institución educa-

\footnotetext{
Proyecto Código 1106-740-54599 financiado por Colciencias.

4 Nombre ficticio para proteger la identidad del menor.
} 
tiva. La familia de Horacio presentó una denuncia penal (22 julio 2014) contra el joven Urrego por aparente acoso sexual del hijo (Corte Constitucional, 2015). Las directivas de la institución citaron del manual de convivencia la prohibición de "las manifestaciones de amor obscenas, grotescas o vulgares" (Semana, 2016), y procedieron a llamar a Urrego y sus progenitores para exigirles tomar "acciones preventivas para evitar futuras decisiones sancionatorias" (Corte Constitucional, 2015 p. 2). Urrego fue obligado a asistir a sesiones con la psicóloga de la institución y a visitar a un psiquiatra. El joven se suicidó el 4 de agosto de 2014 como consecuencia de los sentimientos de culpa y del acoso del que fue objeto por parte de algunos compañeros del colegio y el ente institucional.

La madre del joven Urrego, Alba Reyes, instauró una acción de tutela en representación de su hijo fallecido contra la institución educativa y otros, con el asunto:

El Gimnasio Castillo Campestre, la Secretaría de Educación de Cundinamarca, el Instituto Colombiano de Bienestar Familiar, la Fiscalía General de la Nación y la Comisaría Décima de Familia de Engativá promovieron conductas sistemáticas de discriminación en contra de su hijo, motivadas por su orientación sexual, tanto el proceso disciplinario que se surgió en contra, como con la información que fue difundida con posterioridad al fallecimiento del niño en los medios de comunicación, que favorecieron inicialmente su suicidio y que resultaron finalmente lesivas de sus derechos fundamentales. En segundo lugar, frente a las demás entidades estatales acusadas en la tutela, estima que estas desplegaron una conducta omisiva ante las diferentes denuncias que se realizaron con fundamento en las actuaciones equivocadas de la institución educativa, por lo que incurrieron a su vez, en la violación de los derechos fundamentales del menor de edad. (Corte Constitucional, 2015).

Lo que lleva a la revisión de cumplimiento de garantía de al menos dos de los derechos fundamentales ciudadanos plasmados en la Constitución política de 1991: el derecho al desarrollo de la libre personalidad ${ }^{5}$ y al buen nombre ${ }^{6}$. Además, logró demostrar ante los tribunales que la relación entre Sergio y Horacio era consensuada, como lo evidenciaban las conversaciones de chat entre ambos, y que las psicólogas del colegio tenían conocimiento del tipo de relación sentimental entre estos jóvenes y, por lo tanto, no se trataba de acoso sexual.

El fallo de la Sala Quinta de la Corte Constitucional declaró que Sergio Urrego no tenía tendencias suicidas, y que el comportamiento correspondía al de un joven de su edad. Además, exigió una revisión del manual de convivencia de los colegios colombianos para prevenir el acoso escolar. El fallo se recoge en la Sentencia T-478 de 2015, cuyo asunto "Discriminación por orientación sexual e identidad de género en ambientes escolares; protección del derecho a la igualdad y el libre desarrollo de la personalidad; corresponsabilidades en el desarrollo educativo de los menores de edad" (Corte Constitucional, 2015). A partir de esta sentencia la Corte Constitu-

Artículo 16. Todas las personas tienen derecho al libre desarrollo de su personalidad sin más limitaciones que las que imponen los derechos de los demás y el orden jurídico. (Constitución Política de Colombia, 1991).

6 Artículo 15. Todas las personas tienen derecho a su intimidad personal y familiar y a su buen nombre, y el Estado debe respetarlos y hacerlos respetar. De igual modo, tienen derecho a conocer, actualizar y rectificar las informaciones que se hayan recogido sobre ellas en los bancos de datos y en archivos de entidades públicas y privadas. (Constitución Política de Colombia, 1991). 
cional ordenó revisar los manuales de convivencia de las instituciones educativas e incluir la diversidad sexual y de género para evitar el acoso escolar.

La Ministra de Educación (2014-2016) responde a esta demanda de la sentencia con la firma un convenio con el Programa de las Naciones Unidas para el Desarrollo (Unfpa) y Unicef para editar una cartilla titulada Ambientes escolares libres de discriminación (2016). Allí se recogen algunos lineamientos que el Ministerio brinda a las instituciones educativas como propuesta para afrontar el reto de la inclusión de las diversidades de género y orientación sexual. El público objetivo de la cartilla son rectores y docentes de las instituciones educativas, quienes desde el principio de "autonomía educativa" deciden sobre los mecanismos, dinámicas y recursos humanos y materiales para responder a la demanda constitucional de inclusión de género y sexualidades.

Desde sectores políticos con orientaciones conservadoras y judeocristianas se desarrolla una campaña opositora a esa propuesta. Plazas públicas, iglesias, circuitos cerrados de televisión, radiodifusoras, periódicos, panfletos y redes sociales fueron utilizados como espacios de comunicación para elaborar una propaganda desacreditadora de la cartilla que buscaba sensibilizar desde la instancia escolar a la población frente a temas relacionados con SGD. Se argumentó que el documento constituía una amenaza contra los valores del modelo de familia heterosexual como la legítima.

El 10 de agosto de 2016 miles de padres y madres convocados por la Congresista Ángela Hernández y apoyados por líderes religiosos católicos y cristianos, realizaron marchas y plantones en diferentes lugares públicos para protestar por una supuesta inclusión "de la ideología de género" impuesta a los colegios por MEN". Estos padres y madres de familias reaccionaron indignados y temerosos frente a la amenaza de que sus hijos/as se educaran en libertad de tendencias homosexuales, que atentan contra los principios morales y éticos de familias tradicionales, y exigieron la renuncia de la Ministra.

En la plenaria del Senado de la República nombrada "Debates sobre manuales de convivencia" (16 agosto 2016) la Ministra del MEN argumentó frente a las acusaciones de los opositores que la propuesta era constitucional y que aquella se derivaba del cumplimiento a una demanda planteada por un fallo de la Corte Constitucional. Antes de renunciar al cargo el 2 de octubre 2016, la funcionaria denunció la deshonestidad de ciertos grupos políticos y religiosos al movilizar la opinión pública por medio de mentiras y desinformación.

Paralelo a estos hechos, se desarrollaba en el país un suceso histórico: la discusión y firma de los acuerdos de paz con las Fuerzas Armadas Revolucionarias de Colombia (FARC) (2014-2016). Se inaugura una era de postconflicto para Colombia, lo que significa además del cese al fuego, el replantear un nuevo modelo cultural no-guerrerista y no-violento en una propuesta de construir una cultura del posconflicto por medio de una educación para la paz (Pedagogía para la paz, 2016).

La firma del acuerdo para la terminación del conflicto y la construcción de una paz estable y duradera (2016) respalda lo expuesto en el Decreto 1038 de mayo de

\footnotetext{
Ver reportajes de los periódicos El Tiempo (10 agosto 2016) Asi fue la marcha contra supuestos cambios en manuales de convivencia. Bogotá: El Tiempo. Recuperado de http://www.eltiempo.com/colombia/otras-ciudades/protestas-contra-cartillas-de-ideologia-de-genero-en-colegios-42293 y Guerrero, C. (10 agosto 2016) Los temores detrás de la marcha por la familia. Bogotá: El Espectador. Recuperado de http://www.elespectador. com/noticias/bogota/los-temores-detras-de-marcha-familia-articulo-648506
} 
2015 del MEN, que exige a todas las instituciones impartir las llamadas "Cátedras de paz", las cuales se comprometen con la inclusión de las diversidades (raza, clase, preferencia sexual, étnica, de género), la prevención de la violencia y el respeto por los Derechos Humanos. Este compromiso asumido desde el 2015 sirve como fundamento a la demanda de la Corte Constitucional de revisión de manuales de convivencia de las instituciones educativas en materia de garantía de opciones para la vivencia de SGD como evidencia del libre desarrollo de la personalidad.

La reflexión sobre la construcción de espacios alternativos para la inclusión de las SGD en la escuela a través de los MC se realiza en un diálogo interdisciplinario que recurre a aportes de teóricas y teóricos postmodernos feministas y de género, y a contribuciones desde las teorías de la comunicación.

Cuando hablamos aquí de MC nos referimos a una serie de instrumentos culturales utilizados para informar y comunicar, que intervienen en la socialización y las relaciones entre las subjetividades, que conforman los diversos grupos humanos. Desde el punto de vista de la teoría de la socialización en toda interrelación interviene la comunicación, sea esta verbal, no verbal, escrita, audiovisual, entre otras (Sánchez, 2001; González, 2012; Hall, 1997; Téramo, 2006). De allí que autores como Gregory Bateson (1971), Paul Watzlawick (1994), Marcelo Pakman (1996), Stuart Hall (1997), Manuel Castells (2001) o Carlos Scolari (2015) afirmen que los MC se configuran como herramientas que apoyan los procesos del devenir cultural.

A través de los $\mathrm{MC}$ se han producido una serie de redes o "sociedades red" (Castells, 2001) que conectan instituciones, naciones, personas, movimientos sociales y civiles, las cuales en conjunto construyen cultura. Según los autores, los MC permiten configurar identidades, sugieren modos de actuación, construyen imaginarios sociales, proponen formas de socializar y de aprender. Todos estos procesos en los que intervienen los medios de están transversalizados por múltiples categorías sociales como el género, la orientación sexual, la etnia la clase, la raza, entre otras.

Joan Scott (2011) rescata el significado y la validez de la categoría de género en el análisis complejo de los fenómenos sociales. Desde el punto de vista de la autora la categoría de género es históricamente situada, o sea, se corresponde con el mundo ideológico, y materialista del espíritu de cada época. Es así como los MC como instrumentos de trasmisión de información (conocimiento) contribuyen a mantener o innovar ideas, imaginarios y actuaciones acerca de seres de género situados en determinados contextos.

Retomando los aportes de Olga Obando (2013) se asume la subjetividad de género, como esas experiencias de significar los mundos de género en los cuales se vincula cada uno de los seres humanos. La autora reconoce al menos cinco características de las SG: son procesuales, experienciales, diversas, contextualizadas, situadas. En la opinión de la autora las SGD compartirían todas las características.

Desde las perspectivas performativas postmodernas de género (Braidotti, 2002, 2004, 2013; Butler, 2002, 2006; Haraway, 1995; Preciado, 2013) se reconoce la experiencia subjetiva de género como una experiencia discursiva en la cual la actuación reiterativa de actos discursivos (hablados o actuados) se incorporan, negocian y transgreden. Estos actos contienen mandatos sociales culturales y políticos de género. El fenómeno de género supera en esta perspectiva los asuntos de sexo y orientación sexual y se politiza. La característica relacional de la experiencia performativa de género es sensible a las relaciones de poder que se instauran como discursos normativos del cuerpo y las emociones. Al respecto, Butler plantea: "el género se construye 
a través de las relaciones de poder, y específicamente, las restricciones normativas que no solo producen, sino que además regulan los diversos seres corporales" (2002, p. 13), y va más allá del fatalismo determinista discursivo al reconocer a los sujetos de género en su capacidad de agencia. Negociar y transgredir normativas discursivas de género es la opción libertaria y emancipadora de las subjetividades diversas.

¿En qué consiste entonces la diversidad de género? Seguramente el primer aspecto a referenciar que se nos ocurre desde el sentido común frente a esta pregunta es el referido a la orientación sexual de los sujetos, que en clave normativa hegemónica se refiere a todo aquello que difiere de los cánones heterosexuales, pero lo diverso en el género se construye en la transversalidad con otras categorías que han funcionado como características sociodemográficas de las poblaciones. El abordaje complejo del fenómeno de género reconoce esa heterogeneidad que emerge de la interrelación de factores y características de lo humano, esa que va más allá del determinismo biológico de lectura sesgada, en encuadre dual, que limitó los aportes de conocimiento sobre la diversidad humana producidos desde las ciencias biológicas, como ciencias de las biodiversidades.

Asumir una perspectiva que reconoce la diversidad de género implica ampliar la comprensión del proceso de "generización" de esa regulación de estereotipos, estéticas, valores y características hegemónicas, heterosexuales, androcéntricas, falocéntricas asignadas como norma a las expresiones de sexualidad y género, superar el mandato de únicas formas del ser de género, como marco de un “deber ser" de una corporeidad enunciada como normal según un determinado sexo. Asumir una posición crítica acerca del entramado en la propia caracterización como sujeto de género.

Partimos del supuesto que los MC como instrumentos que intervienen en el devenir cultural ayudan a la construcción de las subjetividades de género de manera ambivalente, desde la hegemonía o desde la diversidad. Y es precisamente esa ambivalencia de los medios, desde sistemas paradojales lo que pone en tensión las dos posturas tecnofóbicas y tecnofílicas que analizan el significado de los fenómenos comunicativos mediáticos en su relación con la sociedad y la cultura.

Los aportes para comprender el fenómeno desde una perspectiva tecnofóbica ofrecen un acumulado de estudios sobre MC en los que se apunta a considerar, desde un razonamiento de causa-efecto o desde un determinismo cultural, que los medios masivos mercantilizan y alienan a las sociedades sin que los sujetos puedan evitarlo (Lasswell, 1938; Martin, 2002). En estos estudios los MC son considerados como instrumentos de control y condicionamiento, lo que los constituyen en el cuarto poder al servicio del Estado y la empresa privada, que a manera de máquinas de felicidad producen consumidores/as. Estas teorías se basan entre otros en unos postulados marxistas en los que se plantea que "las ideas de la clase dominante son en todas las épocas, las ideas dominantes" (Marx \& Engels, 1976, p. 78). Un ejemplo de posturas tecnofóbicas se desarrolla en la "teoría hipodérmica" de la comunicación (Lasswell, 1938) que indica a modo de metáfora que los MC son como una gran aguja que literalmente inyecta ideologías a esas masas acríticas, que no tienen otra opción que la alienación. Otra propuesta teórica desde la cual se señala el poder opresor de los MC sobre las subjetividades es la "teoría de la hegemonía" (Martin, 2002) que continuó con la línea marxista pero lo hace desde el concepto de hegemonía gramsciana. En esta línea, la cultura se define como una superestructura cuyas normas son impuestas por la burguesía a través de un proceso de naturalización e internalización de la norma impuesta a la clase dominada. A diferencia de la teoría hipodérmica, la con- 
sumidora o el consumidor no necesariamente recibe los mensajes de manera pasiva, aunque termine cediendo a los discursos mediáticos.

A pesar de que una teoría como la hipodérmica es cuestionada, sigue en vigencia para explicar la lógica que subyace a las acusaciones que plantean sectores civiles y políticos al considerar que la cartilla del MEN atenta contra "la moral y las buenas costumbres". El hecho de significar como amenazante y poderoso un instrumento de comunicación legalizó una movilización ciudadana en contra de la propuesta de unos lineamientos para garantizar la inclusión de la diversidad sexual y de género desde el quehacer del contexto escolar.

Algunas autoras feministas asumen posturas tecnofóbicas al analizar los $\mathrm{MC}$ como motores de invisibilización de las minorías, como naturalizadores del "ser femenino", y como medios de cosificación de las mujeres y de los sujetos feminizados según las tendencias del mercado (Agudelo, 1997; Kilbourne, 1999; Valladares, 2012).

Una revisión de productos comunicativos que circulan en las TIC como publicidades y chistes gráficos conocidos popularmente como memes que se evidencian en la Figura 1, otorgarían la razón a los argumentos tecnofóbicos en tanto se recrean tres representaciones de la mujer tradicional como modelo de subjetividad femenina: la mujer como ama de casa, representada en los comerciales de productos de aseo ${ }^{8}$ (Figura 1A); la mujer como objeto sexual erótico, presentada como objeto comercial de insumos para construcción ${ }^{9}$ y de la industria de perfumes ${ }^{10}$ (Figuras 1B y 1C), y en la Figura $1 \mathrm{D}^{11}$, la mujer subvalorada. naturalizando con ello un estatuto de mujer no emancipada de sistemas opresivos culturales, políticos y económicos.

Para el caso del chiste gráfico (meme), el gesto del hombre alude al carácter "incorregible" de las mujeres, imaginario social de corte machista que hace parte de esta suerte de industria de estereotipos de subjetividades generizadas que reiteran un imaginario de la mujer como un ser misterioso, engañoso, objeto de deseo, contradictorio y abrumador (Agudelo, 1997).

Desde las investigaciones tecnofílicas se identifican tres características en los MC: ser democratizantes; ser aliados de la liberación, y ser determinantes en el desarrollo social, económico, político y cultural.

Dichos estudios valoran como una de las potencialidades de los MC, especialmente de las TIC, el poder para constituirse en espacios democratizantes y aliados de la liberación, debido a la capacidad que tienen los nuevos medios para alojar y hacer circular información y a la imposibilidad de los sistemas para fiscalizar todo los conocimientos, saberés y prácticas que fluyen virtualmente a través de esos espacios. Es así como la libre expresión encuentra un espacio casi ilimitado de acción. Sin embargo, estas posturas analíticas tienden a minimizar aspectos como la censura, ciberpersecusión y el panóptico mediático (Feinmann, 2013). Además, se parte del supuesto que el sistema-mundo está verdaderamente globalizado y conectado a través de internet, argumento que se relativiza frente los datos del Banco Mundial (2015) que evidencian que solo un $44 \%$ de usuarios/as del mundo se encuentran conectados a internet, y para el caso de Colombia solo se registran un $55,9 \%$ de usuario/as de este medio.

\footnotetext{
https://infogram.com/estereotipos-y-roles-de-genero-en-la-publicidad-televisiva-1gg4qpz69q6521y

http://www.tarata21.com/2015/01/vania-bludau-y-la-polemica-campana.html

https://blogs.ubc.ca/jinajeong/2016/02/26/grsj-300-culture-jam-tom-ford-and-boob-marketing/

http://www.memegen.es/meme/x5ivnv
} 


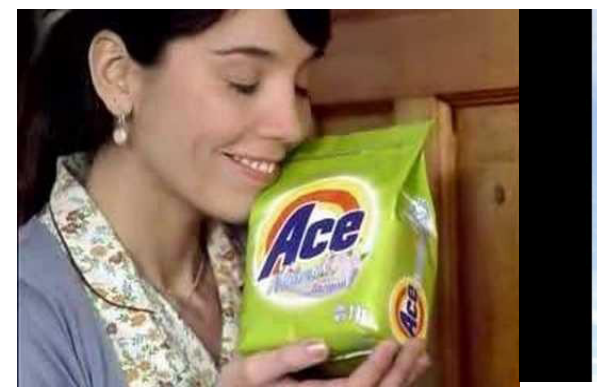

A

$\mathrm{C}$

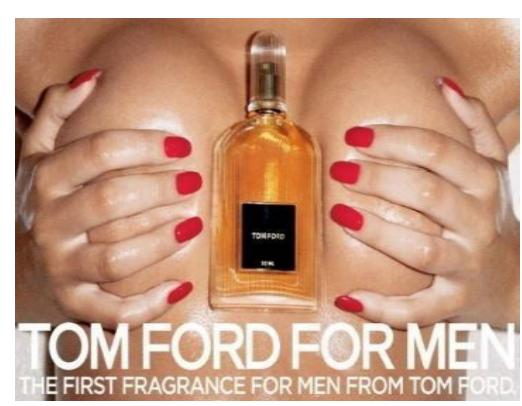

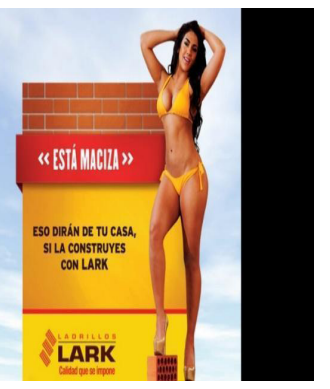

B

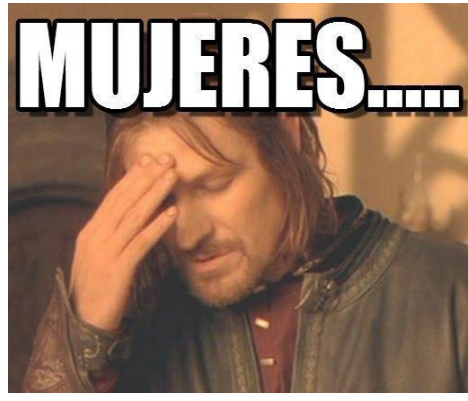

$\mathrm{D}$

Figura 1. Imágenes A, B, C corresponden a representaciones femeninas hegemónicas de publicidades en medios de comunicación. La imagen D es un ejemplo de este tipo de representación en chiste gráfico (meme).

Desde un corte tecnofílico feminista y de género, algunas autoras feministas apuestan por incluir los avances tecno-científicos al servicio de la causa emancipatoria (Wajcman, 2008; Braidotti, 2002; Pérez-Bustos, 2010; Reverter Bañón, 2013; de Miguel \& Boix, 2002). Es el caso temprano de la feminista radical Shulamith Firestone quien plantea en La dialéctica del sexo (1976) una postura tecnofílica al afirmar que si la raíz de la opresión se encuentra en la "servidumbre biológica" de la mujer, la liberación estaría en técnicas de reproducción artificial. Donna Haraway (1995) identifica a la tecnología como potencial aliada en la estrategia política de unir las resistencias de diversos grupos minoritarios históricamente oprimidos, hoy globalmente interconectados por las TIC. La Figura 2 presenta algunos ejemplos de expresiones feministas en los $\mathrm{MC}^{12,13}$ en los cuales se plantea en un vocabulario mediático de la TIC, los objetivos políticos del ciberfeminismo frente al patriarcado o la intención de las mujeres de expandir su apropiación de los espacios públicos como escenarios políticos.

Pierre Lévy considera que el ciberespacio, representado en la plataforma internet, es un espacio potente para el actuar político de las colectividades locales. Para el autor:

https://soyunachicamala.wordpress.com/2015/10/07/ciberfeminismo/

13 http://miriamherbon.com/ciberfeministas-estan-en-todas-partes/ 
La verdadera democracia electrónica consiste en animar tanto como es posible gracias a las posibilidades de comunicación interactiva y colectiva ofrecidas por el ciberespacio- la expresión y la elaboración de los problemas de la ciudad por los ciudadanos mismos, la autoorganización de las comunidades locales, la participación en las deliberaciones de los grupos directamente concernidos por las decisiones, la transparencia de las políticas públicas y su evaluación por los ciudadanos. (2007, p. 158).
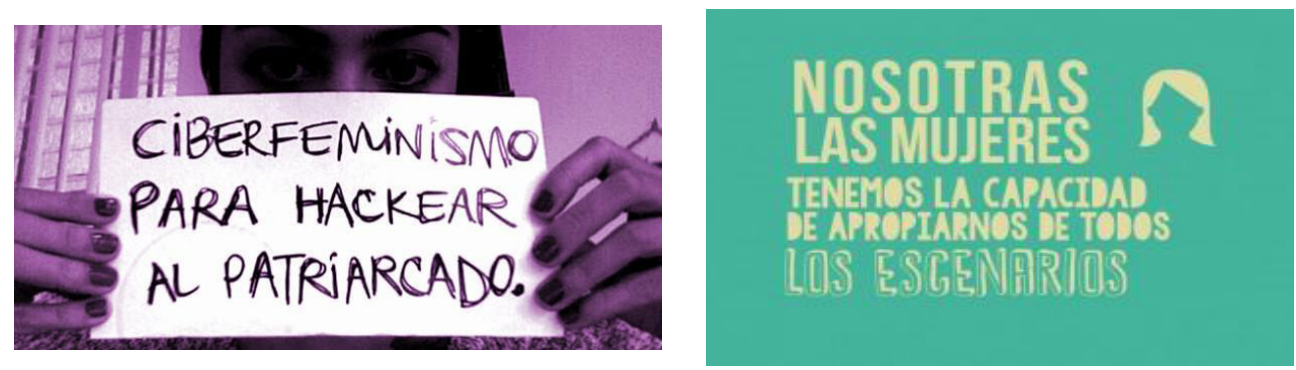

Figura 2. Campañas ciberfeministas que circulan en internet.

Esta posibilidad de construir espacios democráticos en plataformas digitales es un trabajo que está produciendo Gonzalo Frasca (2001) desde otro sector de la industria de las comunicaciones y el entretenimiento: los videojuegos. Para este autor es posible catapultar el videojugar como una experiencia que derive en concienciación de las opresiones sociales y con ello, la apertura a la reflexión, el pensamiento crítico y debate político. Frasca, basado en la teoría del teatro del oprimido de Augusto Boal, considera que es posible diseñar videojuegos del oprimido que produzcan modelos mentales propositivos para la configuración de sujetos políticos.

Algunos autores al analizar los MC introducen otras categorías como la resistencia, la identidad, la raza, la etnia, la clase, la edad y el género lo que permite nutrir una comprensión más informada de los MC desde la complejidad de los mismos (Gómez, 2013; Soley-Beltrán, 2015; Haraway, 1995; Braidotti, 2002; 2013; Hall, 1997). Ejemplo de ello son los análisis que realiza Stuart Hall desde la Escuela de Birmingham" al abordar el papel de los medios como mediadores de la cultura popular y las emergentes "subculturas" juveniles.

Las posturas tecnofílicas, si bien son sensibles en hallar en los MC espacios de democratización, rupturas de la norma y centros de confluencia política, parecieran minimizar el origen de estas plataformas y las paradojas que allí se gestan. En la propuesta de inclusión revisada, el MEN actuó con ingenuidad al suponer que el trabajar con equipos expertos en la elaboración instrumento mediático para la propuesta, le garantizaría el éxito de la misma y al obviar el entramado de intereses (significados e imaginarios) desde los que se leería la propuesta y se movilizaría la oposición a la misma.

Para evitar esta dicotomía de interpretar los medios acorde a sus efectos negativos y/o positivos y ampliar los marcos de análisis de los significados, a unos que permitan comprender su potencial para actuar frente a los fenómenos de inclusión y exclusión de SGD, se hace necesario interpretar a los MC como sistemas complejos.

De acuerdo con Edgar Morin (1999; 2004) el paradigma de la complejidad relaciona la teoría general de sistemas, la teoría de la cibernética, la teoría de la au- 
toorganización y las teorías de la comunicación de Gregory Bateson (1971), Paul Watzlawick (1994), Marcelo Pakman (1996). Todas estas teorías de diversos campos del conocimiento permitieron a Morin, señalar que para comprender los fenómenos es necesario hacerlo desde múltiples campos del conocimiento. El principio epistemológico de este paradigma es la incertidumbre (Morin, 2004), lo que lo distancia del determinismo biológico y/o cultural. Este paradigma vincula la implementación de las TIC como un fenómeno que dinamiza la construcción del conocimiento y que fortalece diversos tipos de aprendizajes como los significativos, contextualizados y transdisciplinarios. Para Hilbert (2011), las características de los sistemas complejos son: conectividad, interdependencia, diversidad, adaptabilidad, sensibilidad a las variaciones y tempo-dependientes, inestabilidad, emergencia y caóticos.

Desde una óptica feminista y de género, Donna Haraway (1995) y Rosi Braidotti (2013) coinciden en la imperiosa necesidad de incluir en los análisis de fenómenos sociales contemporáneos un paradigma de la complejidad que incorpore los análisis críticos de género a las tecnosociedades contemporáneas. Asimismo, estas autoras convergen en comprender a los sujetos desde el devenir, la contradicción, el dinamismo, el contexto situado y la diversidad, distanciándose así de la idea de un sujeto esencial y universal.

Con base en la perspectiva de la complejidad los MC no son en sí mismos máquinas opresoras y alienantes, ni tampoco ventanas de liberación. Estos se significan como espacios producidos y productores de sociedad y cultura. Los MC responden y reflejan procesos del devenir cultural al vincular: la sociedad con sus instituciones, la producción de expresiones culturales y las escuelas (formales y no formales). De esta vinculación se gestan espacios de construcción de identidades diversas, apoyadas en la transformación/transgresión de saberes a partir de posiciones ideológicas diversas.

Comprender los MC como unos sistemas complejos que pueden ser leídos como indicadores de tendencias culturales y como espacios-tiempos en donde se continúa el aprendizaje (Gros, 2015) permite poner en tensión las lecturas clásicas de aprendizaje formal que han reducido las experiencias de adquirir conocimiento a las vivencias en el aula de clase. De allí que sea necesario que académicos, docentes, instituciones, estudiantes y otros actores que intervienen en el proceso educativo centren las discusiones en la comprensión de los contenidos de aprendizaje que se presentan y en los procesos de aprendizaje que se generan en los MC dentro y fuera del aula.

Si retomamos el caso de la cobertura mediática desplegada con relación a lo propuesta de inclusión de género del MEN no es suficiente con centrarse en lo sucedido con la marcha de la familia del 10 de agosto del 2016, tendrían que vincularse a los análisis críticos la exigencia de la Corte Constitucional a las escuelas colombianas, los imaginarios sociales existentes sobre la población LGTBi, los intereses políticos de grupos religiosos y partidos conservadores, el desconocimiento general que existe sobre el enfoque de género, así como las demandas que plantea la construcción de una cultura para la paz duradera, basada en el respeto y el reconocimientos de las diferencias y las diversidades pilar fundamental de la era de postconflicto, entre otros. 


\section{Estrategias para la inclusión de la diversidad de género en los Medios de Comunicación}

Los alcances y limitaciones de estas tendencias explicativas para interpretar y comprender el fenómeno de inclusión y exclusión de SGD en el espacio escolar señalan la manera como los MC diseñan algunas estrategias como: la diversificación y naturalización de imaginarios de género, la implementación de una oferta de conocimiento informado y la motivación de procesos de toma de conciencia de género que permiten potencializar procesos para el abordaje de subjetividades de género en contextos escolares y otros contextos sociales.

La estrategia de diversificación que consiste la ampliación de los discursos de género en los formatos de los MC. Al proponer, enunciar y visibilizar formas otras de ser de género es posible producir y recrear modelos alternativos de género encarnados en personajes, estéticas, formas de ser, cuerpos, opciones profesionales, roles, entre otros.

En los procesos sociales opera una estrategia de normalización de género que legaliza la exclusión, la desigualdad y la descripción del mundo desde una mirada patriarcal, universalista y hegemónica (Braidotti, 2004; Butler, 2006; Preciado, 2013). La sociedad colombiana, como heredera de un colonialismo europeo (Curiel, 2007), se rige por los principios eurocéntricos y universalistas para determinar quiénes son "normales" y se ajustan a los cánones de cuerpos blanqueados, sanos, heterosexuales. Sin embargo, estos cánones de normalidad se han ido adaptando a las demandas del mundo contemporáneo neoliberal-globalizado (Amorós, 2005; Connell, 2004; Fraser, 1997).

Los MC han funcionado como difusores de un imaginario de ciudadanos/as del mundo (Giddens, 2000; Connell, 2004) que viajan a donde quieran, compran lo que quieran y hacen virtualmente lo que les apetezca. Estas aparentes subjetividades globalizadas (Connell, 2004) se construyen y se representan a partir de valores y prácticas culturales hegemonizadas, unificadas, serializadas, que de acuerdo con Nancy Fraser (1997) se disfrazan en un supuesto "multiculturalismo" que ocultan procesos discriminatorios como el racismo, sexismo, clasismo, edadismo y otras formas de exclusión que gestan desigualdades sociales. La aparente ciudadanía global se restringe a ciertos grupos de ciudadanos/as occidentales de clase media y alta que logran romper fronteras, y la cultura global unificada los mide desde los cánones, valores y tradiciones de esta élite. Paradójicamente este "multiculturalismo" globalizado también permite evidenciar y transformar valores culturales, porque la consumidora y el consumidor son un pilar fundamental de la evolución de los mercados (da Silva \& Pasa, 2010). De allí que la apuesta -y reto- contemporáneos de educar para la emancipación de género incluya el educar para construir subjetividades conscientes de lo que se consume, que exijan no solo calidad sino sustentabilidad, economía justa y respeto al otro en su diversidad (Galvis \& Obando, 2016).

Pensar en ampliar la discursiva del género hacia una de la del postgénero en términos de Rosi Braidotti (2004) podría ser clave para plantear representaciones de género en los medios masivos que vayan más allá de la diferencia sexual. Un ejemplo de cómo funciona la estrategia de diversificación en la industria del género es el fenómeno de la familia transversalizado por el fenómeno del género. Podríamos traer a colación el replanteamiento del concepto familia que uno de los comerciales de Coca Cola representa ${ }^{14}$ : a partir de unas preguntas que plantean un grupo de niñas

14 https://www.youtube.com/watch?v=UK7UggbL5XY 
y de niños a sus acudientes respecto a la validez del grupo familiar al que pertenecen se evidencian otras posibles formas de ser de la estructura familiar. Así, en este comercial se valida la existencia tanto la familia compuesta por una mujer sin pareja permanente con hijo, como la constituida por una pareja homosexual, entre otras configuraciones, lo que complejiza la noción de familia sin negar la validez de la "familia tradicional". Esta campaña además la respalda una marca de alta recordación y penetración en el mercado, que en principio podría generar fácilmente grados de complicidad por parte de sus consumidores.

Existe una relación entre las estrategias de naturalización y la de diversificación. Una vez que los diferentes MC han incluido en sus formatos y productos una ampliación de discursos de género, se sucede el proceso de naturalización, el cual se gesta a partir de la reiteración de discursivas emergentes. Esta estrategia se materializa, por ejemplo, la presencia en comerciales para sectores invisibilizados (LGTBi, mujeres, grupos racializados, etc.), marketing inclusivo, y la inclusión de personajes con modelos que amplían la pluralidad del género.

Para el caso de la construcción de las SGD es necesario que las usuarias y los usuarios en las diversas etapas vitales estén en contacto con identidades de género que incluyan el ser y sentirse diferentes a la norma de género hegemónica y con ello naturalizar otras formas de expresión de género. Esta policromía de subjetividades se nutre de las luchas a favor de la reivindicación de los derechos de los grupos históricamente oprimidos, y formas diversas de vivenciar las subjetividades en grupos de mujeres, hombres, poblaciones racializadas, jóvenes, niños/as o el de la población LGTBi, las cuales van minando la hegemonía de un único modelo sujeto universal y única forma de subjetividad de género. Paralelamente los mercados, impulsados por las demandas de los clientes emergentes de estos sectores sociales, se ven obligados a ampliar el espectro de oferta de bienes y servicios que se verán reflejados en los $\mathrm{MC}$.

Ejemplos de ello son publicidades como las de Doritos ${ }^{15}$ o Tiffany co. ${ }^{16}$, que apuestan a ampliar los mercados a un público objetivo LGTBi (Ver Figura 3). Para el caso de la marca de comestibles, su empaque hace alusión a la bandera del orgullo gay, icono que se encuentra en el imaginario cultural y que permite posicionar el producto al nuevo sector objetivo de mercado a la vez que naturaliza la imagen del símbolo gay. Para el caso del anuncio de la joyería, abrir la oferta de anillos de compromiso a una pareja homosexual ayuda a naturalizar la inclusión de esta población históricamente excluida a comprometerse en matrimonio, rito que tradicionalmente está reservado para parejas heterosexuales.

http://www.thedrum.com/news/2015/09/18/need-know-us-marketing-media-news-doritos-lgbt-push-applepay-primes-china

16 https://www.expoknews.com/tiffany-co-lanza-nueva-campana-con-pareja-gay/ 


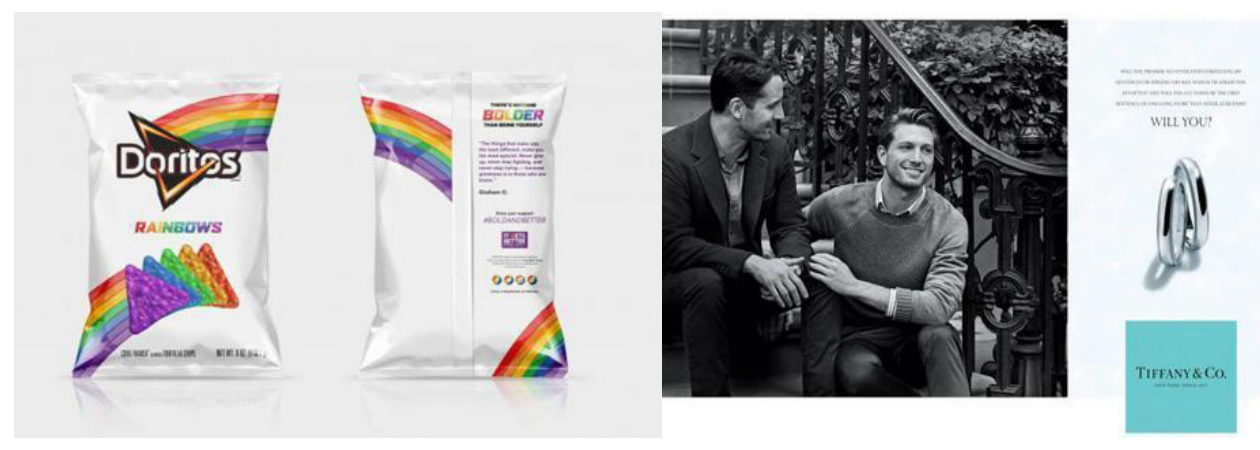

Figura 3. Publicidad dirigida al mercado LGTBi.

También podríamos señalar como ejemplo la apuesta del videojuego Dragon Age: Inquisition (2014) cuyo personaje Krem, abiertamente transgénero, habla de la diversidad de género como un aspecto normal en una dinámica social. Asimismo en los videos "soy princesa siendo yo" 17 y "Kotex: no te detengas"18 que amplían las posibilidades de acción de los sujetos generizados en los espacios públicos y privados.

El conocimiento informado como estrategia de inclusión de SGD se refiere a la posibilidad de producir saberes con sustento teórico que permita a los usuarios incorporar en sus discursos argumentaciones a favor de la diversidad.

Los MC, especialmente las TIC, han democratizado la información (Lévy, 2007). Evidencia de ello es que virtualmente cualquier usuario/a -niño/a o joven para el caso de la población mayoritaria del sector educativo- que se conecte a la red puede acceder a miles de plataformas que brindan información de diferentes calidades. Lo que favorece el acceso a un conocimiento más informado sobre los fenómenos o temas de interés. Este tipo de acceso que rompió con un modelo educativo moderno al descentralizar la información que se tenía sobre el mundo de lugares y agentes específicos. De acuerdo con el reportaje de CNN en español (Wallace, 2015) las y los adolescentes y preadolescentes de hoy, en términos globales, interactúan con los diversos MC como videojuegos, televisión, internet, libros, cine, entre otros, un promedio de 9 horas diarias, y experimentan procesos de educación y socialización.

Para María Teresa Téramo (2006) la socialización en la sociedad contemporánea ya no se sucede exclusivamente en la familia, escuela o grupo de pares, sino que se ha ampliado a los MC. Para el caso argentino, según los estudios realizados por la Comisión Federal de Radiodifusión en el 2004 "la cantidad de horas promedio que un menor ve televisión en Argentina supera anualmente la cantidad de horas diarias de escuela" (Téramo, 2006, p. 90).

En la opinión de Begoña Gros existe una relación entre MC y los procesos educativos "Los espacios de aprendizaje están formados por una red en la que se combinan los espacios físicos y los espacios virtuales fortaleciendo la idea del aprendizaje en cualquier momento y en cualquier lugar". (Gros, 2015, p. 59)

Esta conectividad cognitiva personas/máquinas emerge como un tema de interés académico con desarrollos teóricos al respecto (González, 2012; Frasca, 2001; Morin, 1999; Gros, 2015). Desde la teoría del aprendizaje ubicuo "Los dispositivos digi-

https://www.youtube.com/watch?v=NwDh4b3kAgc

18 https://www.youtube.com/watch? $\mathrm{v}=$ LWtspsN80UA 
tales facilitan la ubicuidad, nos acompañan, forman parte de nosotros y constituyen artefactos cognitivos y emocionales de gran relevancia para el aprendizaje (Gros, 2015, p. 59). Siguiendo a Bill Cope y Mary Kalantzis (2010) las características de este tipo de aprendizaje son: permanente, accesible, inmediata e interactiva. En este sentido, los MC como las TIC se convierten en un espacio-tiempo extensivo para aprender y (des)aprender (Frasca, 2001; 2012; Lacasa, 2011; Gros, 2015; Castells, 2001; González, 2012).

Espacios de acceso a conocimiento informado a disposición son: bases de datos, repositorios tipo Redalyc, páginas de bibliotecas, páginas web de instituciones que trabajan con temas de género, redes sociales, comunidades virtuales, videojuegos, páginas Web, Blogs, entre otros, en donde se alojan materiales audiovisuales, infografías, literatura especializada que devela otras experiencias y mundos de género y presentes en actitudes, identidades, expresiones de género y sexuales que superan la oferta normalizada de dualidad heterosexual o expone posibles contravías con lo aceptado social y culturalmente como correcto para una determinada subjetividad de género.

La toma de conciencia de género demanda un proceso que incluye acciones para visibilizar, crear condiciones de enunciabilidad poner en cuestión y romper con las teorías y posiciones hegemónicas (Obando, 2013; Galvis \& Obando, 2016). Consideramos que la concienciación sobre las opresiones que sujeta a la subjetividad sigue siendo un motor de cambio social y cultural. Judith Butler (2006) plantea que en la medida en que la gente sea más consciente de las opresiones que lo subjetivan, como el caso de la norma de género, es posible hacer transformaciones de esa norma coercitiva que normaliza cuerpos basado en un dato biológico conocido como "sexo".

Algunos productos de denuncia crítica como los chistes gráficos (memes), campañas de equidad / igualdad de género o propagandas de inclusión de diversidades son espacios que permiten a los usuarios reflexionar sobre asuntos referidos al género y la diversidad de género.

Un ejemplo del impacto que puede tener el contenido sobre SGD como implícito en un producto de las TIC es el video de la youtuber Miare's Project "Un menor transexual se suicida por culpa del acoso"19. En este medio la youtuber aborda subjetivamente el tema del suicidio de una chica trans barcelonesa por ciberbullying y con ello invita a reflexionar sobre expresiones de SGD. Otros videos institucionales que abordan a modo de campañas de sensibilización social asuntos sobre el sexismo y la discriminación de género son los casos de: "correr como niña" ${ }^{20}$ y el video "Mujer se disfraza de hombre para jugar futbol con los hombres y dejarlos con la boca abierta" ${ }^{21}$. En el primer video se entrevistan a varias niñas, jóvenes y mujeres deportistas con el objetivo de reflexionar sobre la existencia de discursos sobre prácticas expresivas corporales generizadas y jerarquizadas que subyacen en expresión discriminatoria "como una niña". En el segundo video, disfrazan a una mujer futbolista de hombre para jugar de incógnito en un partido de fútbol masculino y tras ganar el juego, la jugadora revela su identidad de género para captar reacciones de sus compañeros. El fin de este video es reflexionar sobre formas de relaciones generizadas en prácticas deportivas a las que les subyacen imaginarios culturales sobre destrezas físicas masculinizadas.

\footnotetext{
https://www.youtube.com/watch?v=gwOfuNZxdX4

https://www.youtube.com/watch?v=s82iF2ew-yk

https://www.youtube.com/watch?v=nf-ry6p4jxw
} 
Los videojuegos aportan a la estrategia de inclusión de la diversidad, de manera explícita. Se resaltan las apuestas que visibilizan y enuncian diversidades de géneros como el caso de Dys4ia (2012), una propuesta indie ${ }^{22}$ que representa una versión de la experiencia de un cuerpo transgénero que no encaja en la sociedad. La Figura 4A ofrece una representación del sentir de género en el videojuego. De forma implícita, y desde las experiencias del usuario/a, un laboratorio de expresiones como Second Life permite a los/as usuarios/as encarnar múltiples cuerpos. Conforme se adquiere mayor nivel de apropiación tecnológica (aprendizaje) mayores son las posibilidades de customizar en los avatares construidos esa cibercorporalidad que se está encarnando y con ello, la customización de expresiones e identidades de género diversos a la manera como lo muestra la Figura 4.

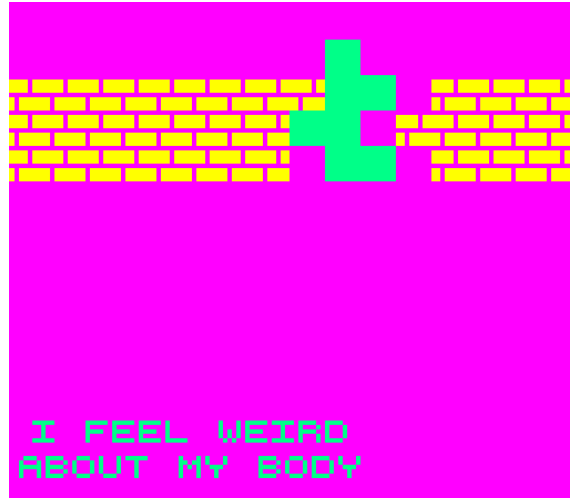

A

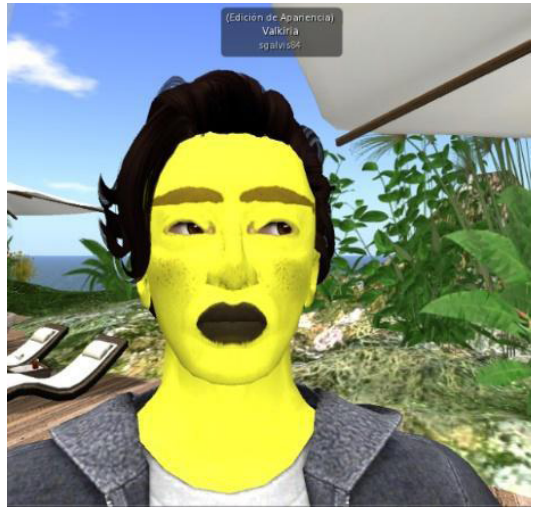

B

Figura 4. A. Representación del sentir transgénero en el videojuego Dys4ia (2012). B. Customización de un avatar de Second Life resultado de tesis doctoral de Sara Galvis (2017).

\section{Inclusión de la diversidad en el sector educativo y ciudadano}

El grado de "autonomía" que poseen las instituciones para el desarrollo de sus proyectos educativos institucionales y modelos pedagógicos se constituye en otro elemento de la complejidad para la estrategia de la inclusión de la diversidad en las escuelas. Dicha autonomía no exime a las instituciones de actuar acorde con los cánones constitucionales y de los principios internacionales de respeto a los Derechos Humanos. Aunque se genere un nodo de tensiones entre la autonomía de las instituciones educativas, los lineamientos del MEN y algunos principios sociales y culturales actuales. Los acontecimientos que se desencadenan como reacción al caso del joven que en el 2014 optó por el suicidio para escapar del acoso por orientación sexual es un ejemplo de ello (Corte Constitucional, 2015). A pesar de que el colegio tenía un manual de convivencia que fue construido autónomamente, en el mismo se desconocía el derecho constitucional de las minorías sexuales a un libre desarrollo

22 El término indie se refiere a producciones alternativas o independientes a las tendencias que determinan el mercado. 
de la personalidad, y de manera indirecta al derecho de las niñas y de los niños a una educación para la diversidad.

La inclusión de la diversidad de género y sexual en nuestra cotidianidad no es un asunto de cumplimiento de lo "políticamente correcto", sino que busca garantizar un pacto de convivencia ciudadana bajo los parámetros de reconocimiento y respeto de las diversidades para promover una cultura de paz estable y duradera en Colombia. En los procesos actuales de construcción de una paz el género se perfila como un eje transversal a los seis principios del acuerdo firmado entre el Gobierno y las FARC (Oficina del Alto Comisionado para la Paz, 2016).

Según el grupo Pedagogía para la Paz (2016) los MC también tienen responsabilidad en la divulgación y comprensión de los acuerdos de paz para que el proceso tenga éxito. El enfoque de género, inserto en los acuerdos es sometido al debate político por actores que representan una diversidad de posiciones ideológicas. La sensibilización hacia estos temas de género, así como la educación sobre lo que significa un enfoque de género, no es una labor exclusiva de las instituciones o de las familias, sino que la interpretación de las informaciones conocimientos y prácticas que involucran de manera directa e indirecta a los MC en la responsabilidad como transmisores de conocimiento.

Todos los actores que intervienen en los procesos de educación de un ciudadano deberán asumir el reto de comprender la realidad contemporánea de género como un fenómeno complejo, que debe ser abordado en el respeto de dicha complejidad, al atender sus entramados y redes de relaciones que allí se tejen.

Otro elemento de la complejidad de la inclusión de la diversidad de género y sexual en el sistema educativo colombiano es la coexistencia de la variedad en los principios ideológicos, éticos, morales con fundamentos diversos los que guíen las instituciones públicas y privadas en el país. Por ejemplo: instituciones con principios y valores conservadores y judeocristianos con posiciones adversas a las discusiones sobre temas sobre diversidad de género y sexualidades. En tanto consideran que ponen en peligro principios morales y éticos, así como instituciones. Por otro lado, aquellas que intentan ser sensibles a incorporar el enfoque de género, pueden caer en esquemas culturales binaristas y patriarcales porque no cuentan con acompañamiento de asesores en el tema. Las instituciones escolares por lo común no logran transversalizar los materiales, las cátedras y el PEI una perspectiva crítica sobre el género.

Es posible mitigar estos vacíos a través de la búsqueda de materiales desarrollados con perspectiva crítica de género en los MC. Ejemplos de estos materiales son la página web española Educar en igualdad ${ }^{23}$ que divulga recursos educativos para la igualdad y la prevención de la violencia de género. Desde una mirada de la equidad de género, se puede señalar la página web chilena Masculinidades y equidad de género $^{24}$ (Ver Figura 5A) y la cartilla impresa diseñada por Unicef Uruguay (2012) titulada Es parte de la vida. Material de apoyo sobre educación sexual y discapacidad para compartir en familia (Ver Figura 5B), en el cual, similar a la propuesta hecha desde MEN, busca orientar a las y a los docentes y miembros relacionados con la educación a comprender asuntos como la educación sexual, diversidad sexual y género y discapacidad. Por último, podemos incluir materiales didácticos que aportan a la inclusión de otros modelos de ser de género como la propuesta argentina

http://www.educarenigualdad.org/material

24 http://www.eme.cl/materiales-educativos/ 
Colección antiprincesas ${ }^{25}$ (Ver Figura 5C), que incorpora otros modelos femeninos construidos por mujeres reales y locales en un intento de ampliar el espectro de diversidades en las experiencias subjetivas femeninas latinoamericanas.

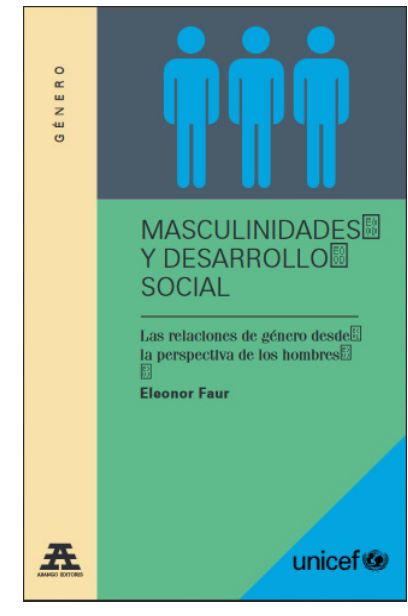

A

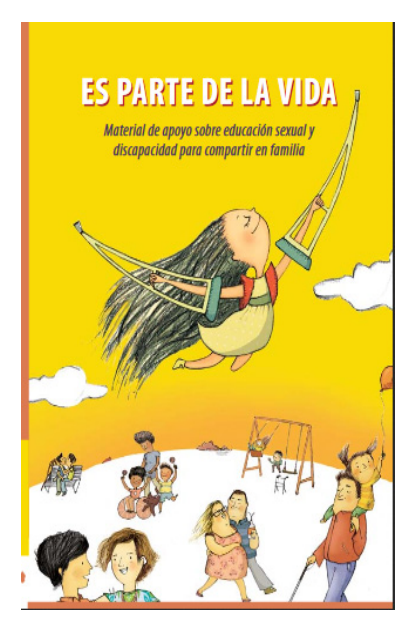

B
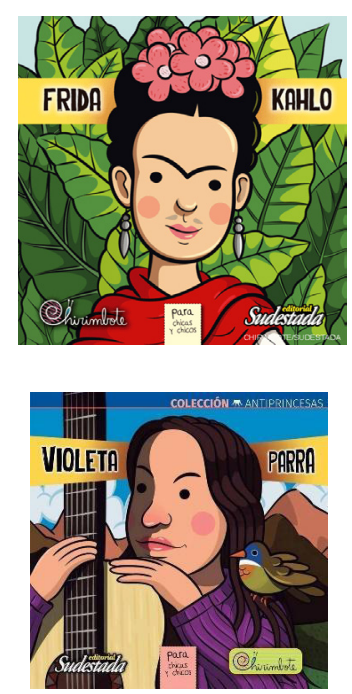

$\mathrm{C}$

Figura 5. Materiales educativos con perspectiva de género.

Las universidades como instituciones educativas, además de insertar en la oferta curricular cátedras de género, impartir charlas sobre salud sexual y reproductiva, deberán abrir y garantizar espacios para la participación de subjetividades que históricamente han sido excluidas y deslegitimadas. En los procesos de construcción de conocimiento generizado se trata de desmitificar la producción y labor académica e investigativa como prácticas masculinas y asumir el reto de visibilizar una historia de patentes, teorías, estudios, producciones en el campo de las ciencias y las artes; aportes realizados por mujeres y hombres de diversas subjetividades de género. Otro reto de las instituciones educativas desde el preescolar hasta la universidad es el de crear estrategias comunicativas internas con el fin de sensibilizar en los temas de género y subjetividades de género al personal docente y administrativo que participa de sus dinámicas.

\section{Conclusiones}

Para responder a la pregunta ¿De qué manera los MC construyen espacios alternativos para la inclusión de SGD en la escuela? se rescatan los aportes de los MC en la oferta de alteración de roles, estereotipos, deseos, cuerpos, estéticas y representaciones de género, alternativos a los hegemónicos, y en la apertura a pensar la existencia de otras SGD diferentes a la propia, a través de campañas comunicativas de

25 http://www.mexiconewsnetwork.com/lifestyle/antiprincesas-books-stereotypes/ 
inclusión de género no sexistas. Incorporar como espacios que oferta la inclusión de las SGD en los medios mainstream que generan megatendencias, naturalizan imaginarios culturales, sociales, políticos actualizantes referidos a la existencia de la SGD al desmontar imaginarios hegemónicos excluyentes. Las redes sociales y medios digitales pueden erigirse como espacios alternativos de expresión de identidades de género y sexualidades diversas. En las instituciones educativas los productos de los MC pueden incluirse como oferta de material didáctico y de textos académicos para visibilizar la diversidad en toda su expresión.

\section{Bibliografía}

Agudelo, M. (1997). Brujas y princesas. Hablemos de comunicación no sexista, 36-42.

Always Latinoamérica (2 julio 2014) ¿Qué significa hacer algo \#ComoNiña?|Always. México D.F.: Youtube. Disponible en: https:/www.youtube.com/watch?v=s82iF2ew-yk

Amorós, C. (2005). Globalización y orden de género. En C. Amorós, \& A. de Miguel (Edits.), Teoría feminista: de la Ilustración a la globalización. De los debates sobre el género al multiculturalismo (pp. 301-332). Madrid: Minerva Editores.

Banco Mundial. (2015). Usuarios de Internet (por cada 100 personas). Disponible en: http:// datos.bancomundial.org/indicador/IT.NET.USER.P2

Bateson, G. (1971). Interacción familiar: aportes fundamentales sobre teoría y técnica. Barcelona: Editorial Tiempo Contemporáneo.

Bigurra, V. (9 marzo 2015) Antiprincesas: Books aimed at fighting stereotypes in children. México D.F.: México News Network. Obtenido de: http://www.mexiconewsnetwork. com/lifestyle/antiprincesas-books-stereotypes/

Braidotti, R. (2002). Un ciberfeminismo diferente. Debats, 76, 100-117.

- (2004). Género y postgénero: ¿el futuro de una ilusión? En R. Braidotti, Feminismo, diferencia sexual y subjetividad nómade (pp. 131-150). Barcelona: Gedisa.

- (2013). The posthuman. Cambridge: Polity Press.

Butler, J. (2002). Cuerpos que importan. Sobre los limites materiales y discursivos del "sexo". Buenos Aires: Paidós.

Butler, J. (2006). Deshacer el género. Barcelona: Paidós.

Campó, S. (7 noviembre 2015). Ciberfeminismo. Obtenido de: https://soyunachicamala.wordpress.com/2015/10/07/ciberfeminismo/

Castells, M. (2001). La galaxia internet Barcelona. Barcelona: Areté.

Connell, R. W. (2004). Género, sexualidad y encarnación mundial: una mirada desde el sur. En M. C. Laverde, G. Daza, \& M. Zuleta (Edits.), Debates sobre el sujeto. Perspectivas contemporáneas (pp. 181-193). Bogotá: Universidad Central - Siglo del Hombre Editores.

Constitución política colombiana (1991). Asamblea Nacional Constituyente, Bogotá, Colombia, 6 de Julio de 1991.

Corte Constitucional. (3 agosto 2015) Sentencia T-478/15. Bogotá: Corte Constitucional. Disponible en: http://www.corteconstitucional.gov.co/inicio/T-478-15\%20 ExpT4734501\%20(Sergio\%20Urrego).pdf

Curiel, R. I. (2007). Crítica poscolonial desde las prácticas del feminismo antirracista. Nómadas, 26, 92-102.

Da Silva, M. E., \& Pasa, C. R. (2010). Consumo consciente: o papel contributivo da Educação. REUNA, 15(3), 43-54. 
De Miguel, A., \& Boix, M. (2002). Los géneros de la red: los ciberfeminismos. El ciberfeminismo social. En R. Baeza, The role of humanity in the information age. A Latin perspective (pp. XX). Santiago de Chile: Universidad de Chile.

Disney Channel LA (18 marzo 2016) Disney Princesa | Soy princesa siendo yo | Puedes ser quién quieras ser. Orlando: Youtube. Disponible en: https://www.youtube.com/watch?$\mathrm{v}=\mathrm{NwDh} 4 \mathrm{~b} 3 \mathrm{kAgc}$

Educar en Igualdad (2017) Recursos educativos para la igualdad y la prevención de la violencia de género. Madrid: Mujeres Fundación. Recuperado de: http://www.educarenigualdad.org/material

El publicista (1 junio 2015) 'Familias', de McCann Erickson Madrid para Coca Cola. Youtube. Recuperado de: https://www.youtube.com/watch?v=UK7UggbL5XY

elizabeth1410 (3 marzo 2017) Estereotipos y roles de géneros en la publicidad televisiva. infogram. Obtenido de: https://infogram.com/estereotipos-y-roles-de-genero-en-la-publicidad-televisiva-1gg4qpz69q6521y

EME (2017) Lista de materiales educativos. Santiago de Chile: Fundación CulturaSalud. Disponible en: http://www.eme.cl/materiales-educativos/

Entretenimiento y Comerciales (24 agosto 2016) KOTEX 'No te detengas'. Lima: Youtube. Disponible en: https:/www.youtube.com/watch?v=LWtspsN80UA

Feinmann, P. (2013). Filosofía política del poder mediático. Buenos Aires: Planeta.

Firestone, S. (1976). La dialéctica del sexo. En defensa de la revolución feminista. Barcelona: Editorial Kairós.

Frasca, G. (2001) Videogames of the opressed: Videogames as a means for critical thinking and debate. (Tesis de maestría). Georgia Institute of Technology, Atlanta.

- (2012). Los videojuegos enseñan mejor que la escuela. TED: Ideas worth spreading. Montevideo: TED. Disponible en: https://www.youtube.com/watch?v=TbTm1Lkm18o

Fraser, N. (1997). Multiculturalismo, antiesencialismo y democracia radical. Una genealogía del impase actual en la teoría feminista". En N. Fraser, Iustitia interrupta. Reflexiones críticas desde la posición "postsocialista”. Bogotá: Siglo del Hombre.

Galvis, S. L., \& Obando, O. L. (2016). Subversión de género en mundos virtuales: algunos lineamientos conceptuales para su producción en Second Life. La Manzana de la Discordia, 11(2), 45-65. doi:https://doi.org/10.25100/lamanzanadeladiscordia.v11i2.1623

Giddens, A. (2000). Un mundo desbocado. Madrid: Taurus.

Gómez, A. (2013). Mujeres y videojuegos. Estudio sobre roles, actitudes y representaciones de las mujeres en los videojuegos. (Tesis de maestría). Universidad Complutense de Madrid: Madrid.

González, J. (2012). Niños que videojuegan, videojuegos que estructuran tiempos: cognición en los bordes del tiempo irreversible. (Tesis de doctorado). Universidad del Valle, Cali.

Gros, B. (2015). La caída de los muros del conocimiento en la sociedad digital y las pedagogías emergentes. EKS, 16(2), 58-68. doi:http://dx.doi.org/10.14201/eks20151615868

Hall, S. (1997). Representation: Cultural Representations and Signifying Practices. (E. Sevillas Casas, Trad.) Londres: Sage Publications.

Haraway, D. (1995). Ciencia, cyborgs y mujeres. La reinvención de la Naturaleza. Madrid: Cátedra.

Herbón, M. (1 marzo 2016) Ciberfeministas, ¿Las conoces? Están por todas partes. Bilbao: Miriam Herbón Ordóñez. Obtenido de: http://miriamherbon.com/ciberfeministas-estan-en-todas-partes/

Hilbert, M. (2011). Charlas sobre sistemas complejos sociales (CCSCS). Una exploración guiada de conceptos y métodos. Online: CEPAL. 
JinaJeong. (26 febrero 2016). GRSJ 300 Culture jam: Tom Ford and Boob Marketing. Jina Jeong's portfolio. Obtenido de: https://blogs.ubc.ca/jinajeong/2016/02/26/grsj-300-culture-jam-tom-ford-and-boob-marketing/

Kilbourne, J. (1999). Can't buy my love. How advertising changes the way we think and feel. Nueva York: Simon \& Schuster.

Lacasa, P. (2011). Los videojuegos. Aprender en mundos reales y virtuales. Madrid: Morata.

Lasswell, H. (1938). Propaganda Technique in the World War. New York: Peter Smith.

Lévy, P. (2007). Cibercultura. Informe al Consejo de Europa. Barcelona: Anthropos.

Martin, J. (Ed.). (2002). Antonio Gramsci. Critical assessments of leading political philosophers. New York: Routledge.

Marx, K., \& Engels, F. (1976). La ideología alemana. México D.F.: Ediciones de Cultura Popular.

McCarthy, J. (18 septiembre 2015) Need to know US marketing \& media news: Doritos' LGBT push \& Apple Pay primes for China. The Drum. Obtenido de: http://www.thedrum. com/news/2015/09/18/need-know-us-marketing-media-news-doritos-lgbt-push-applepay-primes-china

Memegen (2 febrero 2017). Mujeres...- Frustrated Boromir meme. Obtenido de: http://www. memegen.es/meme/x5ivnv

Miare's Proyect (27 diciembre 2015) Un menor transexual se suicida por culpa del acoso. Barcelona: Youtube. Disponible en: https://www.youtube.com/watch?v=gwOfuNZxdX4

Milenio (17 enero 2015). Tiffany \& Co. lanza nueva campaña con pareja gay. Expoknews. Obtenido de: https://www.expoknews.com/tiffany-co-lanza-nueva-campana-con-pareja-gay/

Ministerio de Educación Nacional. (25 de mayo de 2015). Cátedras de paz. Decreto 1038. Bogotá.

Morin, E. (1999). Los siete saberes necesarios para la educación del futuro. París: Unesco.

- (2004). La epistemología de la complejidad. Gazeta de Antropología(20), Texto 20-02.

Obando, O. L. (2013). Luna roja. Herramientas teórico-prácticas para el fortalecimiento de subjetividades de género. Cali: Universidad del Valle.

Oficina del Alto Comisionado para la Paz. (24 noviembre 2016). Acuerdo final para la terminación del conflicto y la construcción de una paz estable y duradera. Bogotá: OACP. Disponible en: http://www.altocomisionadoparalapaz.gov.co/procesos-y-conversaciones/ Documentos\%20compartidos/24-11-2016NuevoAcuerdoFinal.pdf

Pakman, M. (1996). Construcciones de la experiencia humana. Barcelona: Gedisa Editorial. Pedagogía para la paz. (2016). El papel de la comunicación en el postconflicto y la construcción de paz. Obtenido de La conversación más grande del mundo: www.laconversaciónmasgrandedelmundo.com

Pérez-Bustos, T. (2010). Reflexiones sobre una etnografía feminista del Software Libre en Colombia. Revista Estudos Feministas, 2(18), 385-405.

Preciado, B. (2013). Teoría Queer: Notas para una política de lo anormal o contra-historia de la sexualidad. Revista Observaciones Filosóficas, 15. Disponible en: http://www.observacionesfilosoficas.net/queer-teoria.htm

Reverter Bañón, S. (2013). Ciberfeminismo: de virtual a político. Tecknocultura. Revista de cultura digital y movimientos sociales, 10(2), 451- 461. Disponible en: http://teknokultura.net/index.php/tk/article/view/159

Sánchez, Y. (2001). Vygotski, Piaget y Freud: A propósito de la socialización. Enunciación, $6(1), 29-34$. 
Scolari, C. (2015). Ecología de los medios: entornos, evoluciones e interpretaciones. En C. Scolari (Ed.), Ecología de los medios: de la metáfora a la teoría (y más allá). Barcelona: GEDISA.

Scott, J. (2011). El género: ¿Todavía una categoría útil? La manzana de la discordia, 6(1), 95-101. doi:org/10.25100/lamanzanadeladiscordia.v6i1.1514

Semana. (2016). Primera condena por el caso de Sergio Urrego. Obtenido de Semana : http://www.semana.com/nacion/articulo/sergio-urrego-condenan-a-rosalia-ramirez-veedora-del-colegio-gimnasio-castillo-campestre/507458

Soley-Beltrán, P. (2015). ¡Divinas! Modelos, poder y mentiras. Barcelona: Anagrama.

T21 Noticias (11 enero 2015). Vania Bludau y la Polémica Campaña Publicitaria de Ladrillos Lark [Fotos]. Lima: T21 Noticias. Obtenido de: http://www.tarata21.com/2015/01/ vania-bludau-y-la-polemica-campana.html

Téramo, M. T. (2006). Modas adolescentes y medios de comunicación como agentes socializadores. Revista Científica de Comunicación y Educación, 27, 85-91.

Unicef. (2012). Es parte de la vida. Material de apoyo sobre educación sexual y discapacidad para compartir en familia. Montevideo: PES - iiDi - UNFPA - Unicef.

Valladares, C. E. (2012). Análisis del discurso de género en los programas televisivos de producción nacional "Milena tu amiga", "Grandiosas" y "De mujer a mujer", transmitidos de febrero a abril de 2012. Tesis de maestría. Antiguo Cuscatlán: Universidad Centroamericana "José Simeón Cañas".

Vines cali (6 noviembre 2015) Mujer se disfraza de hombre para jugar fútbol con los hombres y dejarlos con la boca abierta. Cali: Youtube. Disponible en: https://www.youtube. $\mathrm{com} /$ watch? $\mathrm{v}=$ nf-ry6p4jxw

Wajcman, J. (2008). Continuidad y cambio. Género y culturas de la tecnología y el trabajo. (TELOS, Ed.) Cuadernos de comunicación e innovación, 74.

Wallace, K. (2015). Los adolescentes pasan 9 horas al día usando los medios, según informe. Nueva York: CNN Español. Disponible en: http://cnnespanol.cnn.com/2015/11/03/losadolescentes-pasan-9-horas-al-dia-usando-los-medios-segun-informe/

Watzlawick, P. (1994). ¿Es real la realidad? confusión-desinformación-comunicación. Barcelona: Editorial Herder. 